\title{
Anterior Percutaneous Endoscopic Cervical Discectomy, a Stitchless and Bloodless Surgery: Clinical and Radiological Results
}

\author{
Sunil M Nadkarni, Sumeet Kautik Sonawane, Satishchandra Gore, Pawankumar Kohli, Muralidhar Varunjikar
}

Departments of Orthopedics and Spine Surgery, B.K.L. Walawalkar Hospital and Rural Medical College, Dervan, Chiplun, Ratnagiri, Maharashtra, India

Corresponding Author:

Sumeet Kautik Sonawane, MBBS, MS Departments of Orthopedics and Spine Surgery, B.K.L. Walawalkar Hospital and Rural Medical College, Flat No. 10, Godavari apartment, Dervan, Chiplun, Ratnagiri, Maharashtra 415606 India

Tel: +917709116165

Fax: +912355264693

E-mail: drsumeet166@gmail.com

Received: August 23, 2018

Revised: November 16, 2018

Accepted: November 28, 2018
Objective: Anterior cervical discectomy with fusion [ACDF] has been considered gold standard for cervical disc herniation over period of time. Anterior percutaneous endoscopic cervical discectomy $[P E C D]$ is minimally invasive technique without need for implant or bone graft in properly selected cases. In this study we present clinical and radiological results of anterior PECD. Methods: We retrospectively studied 31 patients treated with anterior PECD in our institute from January 2014 to December 2016. Patients' clinical data, visual analogue score [VAS], neck disability index [NDI] and radiographs were collected. Cervical lordosis angle, focal angle and disc height of involved segment were measured using Medsynapse software. Statistical analysis was performed using paired T test, chi square test. Results: There were 18 males, 13 females in this study. Mean follow up period was 28.5 months. VAS for neck pain reduced from $6.2 \pm 0.72$ to $1.67 \pm 0.59$; VAS for arm pain reduced from $7.25 \pm 0.71$ to $1.5 \pm 0.61$ at final follow up. NDI reduced from $64.7 \pm 7.62$ to $13.48 \pm 5.42$ at final follow up. Mean disc height of involved segment was $6.15 \mathrm{~mm}$ pre-operatively which reduced to $5.24 \mathrm{~mm}$ at final follow up showing reduction of $0.91 \mathrm{~mm}$. This reduction is disc space is not clinically significant as VAS and NDI show significant fall. Cervical lordosis was well maintained which changed from $13.93^{\circ} \pm 3.7^{\circ}$ to $15.58^{\circ} \pm 6.66^{\circ}$; [p-value is 0.060 ]. Pre-operative focal cervical angle of involved segment was $0.86^{\circ} \pm 1.66^{\circ}$ which increased to $1.7^{\circ} \pm 1.81^{\circ}$ at final follow up [p-value 0.0067 ]. Twenty out of 31 patients resumed their previous employment within 2 weeks. Conclusion: Anterior PECD is excellent minimally invasive technique for cervical disc herniation in properly selected cases. Patients have good functional and clinical recovery after this procedure.

Key Words: Cervical discectomy, Endoscopic cervical discectomy, Minimally invasive spine surgery, Percutaneous endoscopic cervical discectomy, Cervical radiculopathy

\section{INTRODUCTION}

Anterior cervical discectomy with fusion [ACDF] using bone graft or a spacer has been considered gold standard treatment for cervical disc herniation over the period of time ${ }^{1,2)}$. There has been fear that without reconstructing disc space, cervical spine will become kyphotic with distortion of alignment and neural foramen compromise, leading to axial neck pain and delayed radiculopathy. But enough evidence exists that in properly selected cases even without fusion, cervical kyphosis and delayed radiculopathy do not occur and very few cases require delayed fusion ${ }^{3-5}$.

Anterior percutaneous endoscopic cervical discectomy [PECD] preserves majority of anterior and central disc, anterior longi- tudinal ligament, both endplates and facet joints with selective evacuation of offending fragment. This reduces damage to surrounding tissues, bones and ligaments. Thus prevents subsequent malalignment, instability and other approach related complications ${ }^{6)}$.

During this procedure there is minimal blood loss and less muscle trauma. It can be done under local anaesthesia. Hence, patient can be discharged on the next day of surgery reducing hospital stay. There is no need for expensive implants. These factors help in reducing cost of surgery.

Anterior PECD can be performed under local anaesthesia with sedation. Therefore, it can be performed for patients who are not fit for general anaesthesia due to medical reasons. Another benefit of having the patient awake is that the patient can give feedback during surgery which serves like live neuro- 
monitoring.

Anterior PECD has evolved rapidly, but there are very few studies demonstrating its clinical and radiological effects. In this study, we present clinical and radiological outcome of patients treated with anterior PECD using working channel endoscopes and microforceps.

\section{MATERIALS AND METHODS}

\section{Patients}

We retrospectively studied all patients treated consecutively with anterior PECD from January 2014 to December 2016 in our institute. Thirty one patients were operated with anterior PECD during this period. Patients having neck pain and or cervical radiculopathy, soft disc herniation, corroboration of clinical and radiological picture as seen on $\mathrm{MRl}$, failed conservative treatment at least for 6 weeks, disc height more than $4 \mathrm{~mm}$, craniocaudal migration of disc less than half the distance of vertebral body and no previous history of neck surgery were included in this study. Patients having calcified sequestered disc, cervical myelopathy, segmental instability, bilateral symptoms, severe central canal stenosis, no clinic-radiological correlation and history of previous neck surgery were excluded.

Patients' clinical data, pre-operative and post-operative visual analogue score [VAS], neck disability index [NDI] score were collected. Cervical spine AP, lateral, flexion and extension radiographs were obtained. Angle of cervical spine, focal angle and disc height of involved segment were measured using Medsynapse software [Medsynaptic Private Limited, Pune, India].

\section{Statistical Analysis}

Statistical analysis was done using paired T test, Chi square test.

Cervical angle was measured by angle between line drawn parallel to inferior end plate of C2 vertebra and another line drawn parallel to inferior end plate of $\mathrm{C} 7$ on lateral radiograph.

Focal angle of involved segment was measured by angle formed by lines drawn parallel to inferior end plate of cephalad and caudal vertebra. Lordotic angle was designated a positive value while kyphotic angle was designated a negative value.

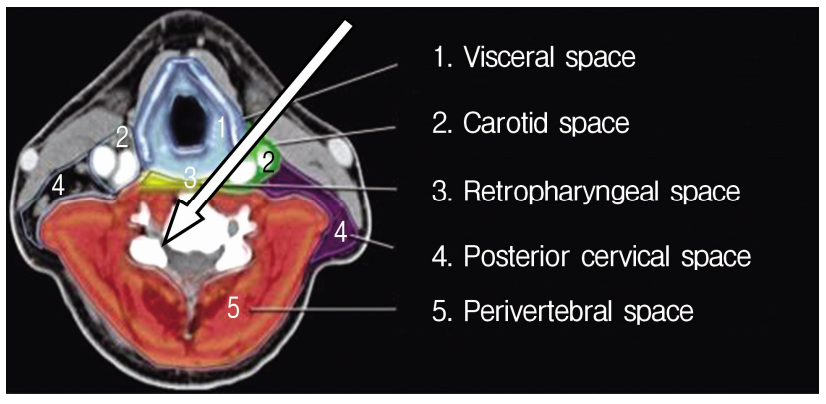

Fig. 1. Trajectory of needle placement.

\section{Surgical Procedure}

Patient was kept in supine position with head ring under head with neck in slight extension, shoulders pulled down, cervical block and sedation was given to minimise pain and anxiety. Approach on neck is taken from opposite side of upper limb having radicular pain. Local anaesthesia $[5 \mathrm{~mL} 0.25 \%$ Bupivacaine $+5 \mathrm{~mL} 2 \%$ lignocaine] was given after marking level under image intensifier.

Compartmentalisation of anatomical structures in neck helps in the approach (Fig. 1). With left hand middle finger, the surgeon displaces trachea and oesophagus towards opposite side and palpates carotid pulse with index finger and displaces it laterally (Fig. 2). This way anterior aspect of vertebral body is reached. Needle with stellate is passed and disc is pierced in midline. This preserves central disc as the trajectory of the needle is oblique and angle depended on position of disc whether central, paracentral or foraminal. Once needle is in disc indigocarmine is injected. Needle is advanced till posterior aspect of vertebral body under image intensifier guidance. Stellate of needle is exchanged with guide wire, 3-5 mm transverse incision is taken, serial dilators are passed and finally camera sheath [cannula] is passed. Its position is confirmed under image intensifier in AP and lateral view (Fig. 3); endoscope [Karl Storz with $2.5 \mathrm{~mm}$ working channel] is inserted through sheath (Fig. 4). Operative space is continuously irrigated with sterile saline which is connected to endoscope.

Using endoscopic forceps and hooks offending fragment is freed and removed. Space for nerve root is restored. Platelet rich fibrin plug was inserted after completion of procedure as there is evidence that it alleviates pain and helps in annular healing $^{7-9)}$. After procedure sterile dressing is applied without stitch.
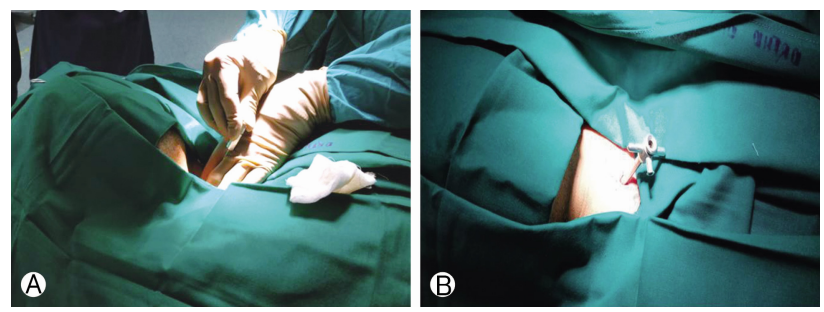

Fig. 2. (A) Needle placement and (B) cannula placement.
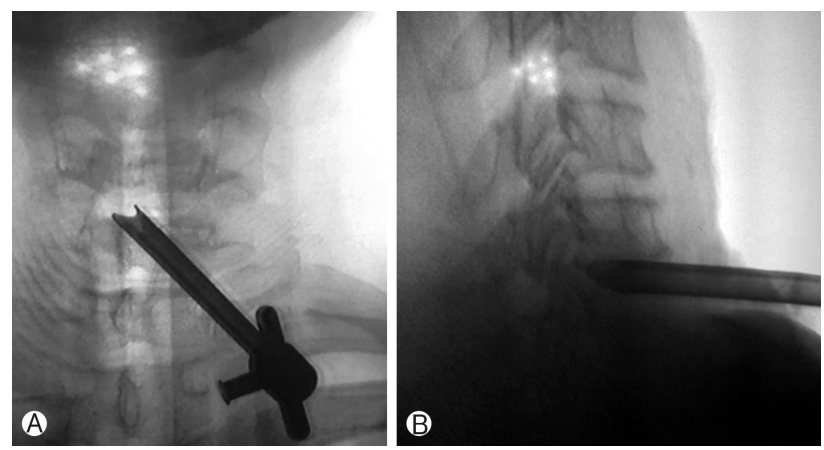

Fig. 3. AP (A) and lateral (B) view after cannula placement. 
This procedure can be performed for $\mathrm{C} 3-\mathrm{C} 4$ to $\mathrm{C} 6-\mathrm{C} 7$ discs. Post-operatively patients were given soft cervical collar and were discharged one day after surgery. Patients were followed up after 10 days, at 6 weeks and every 3 months thereafter. During each follow up, radiographs of cervical spine AP, Lateral, flexion and extension views were taken; VAS, NDI score were recorded. MRI was advised after 3 to 4 of surgery (Fig. 5).

During post-operative and follow up period we were vigilant for complications like no or incomplete relief, neurological deterioration, radicular pain, CSF leak, infections, hematoma formation, dysphagia, delayed radiculopathy, spontaneous or delayed fusion and revision surgery.

This study was approved by our institutional review board.

\section{RESULTS}

During January 2014 to December 2016, a total of 31 patients were operated with anterior PECD. There were 18 males and 13 females. Average age of patients was 38 years, ranging from 25 to 61 years. Mean follow up period was $28.5 \pm 10.1$ months. C3-4 level was operated in 3 cases, C4-5 in 6 cases, C5-6 in 14 cases and C6-7 in 8 cases. Central disc herniation was present in 4 patients 14 had paracentral disc herniation while 13 had foraminal disc herniation.

Pre-operative VAS for neck pain was $6.2 \pm 0.72$ and at final follow up it was $1.67 \pm 0.59$ [p-value $=0.00001]$. Pre-operative VAS for radicular upper limb pain was 7.25 \pm 0.71 and at final follow up was $1.5 \pm 0.61$ [p-value $=0.00001$ ] (Table 1 ). NDI score preoperative was $64.7 \pm 7.62$ and at final follow up was $13.48 \pm 5.42$ [p-value $=0.00001]$ (Table 1).

Mean disc height of involved segment was $6.15 \mathrm{~mm}$ pre-oper-
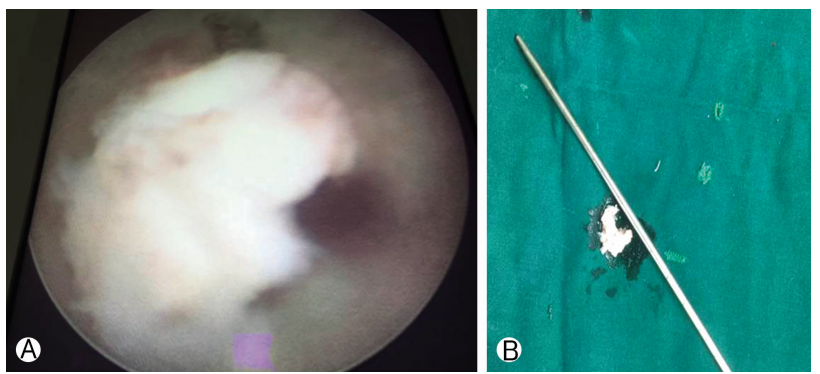

Fig. 4. (A) Endoscopic view of fragment to be removed, (B) removed fragment. atively which reduced to $5.24 \mathrm{~mm}$ at final follow up. There is mean reduction of $0.91 \mathrm{~mm}$ in disc height with $\mathrm{p}$-value $=0.00001$. Though there is reduction in disc height, VAS and NDI also show significant fall which indicate that this reduction in disc height is not clinically significant.

Patients' pre-operative cervical angle improved from $13.93^{\circ}$ $\pm 3.7^{\circ}$ to $15.58^{\circ} \pm 6.66^{\circ}$ at final follow up [p-value is 0.060 ] and overall lordotic angle was well maintained.

Pre- operative focal cervical angle of involved segment was $0.86^{\circ} \pm 1.66^{\circ}$ which increased to $1.7^{\circ} \pm 1.81^{\circ}$ at final follow up which is statistically significant [p-value 0.0067]. There was no segmental instability noted on flexion extension radiograph. These results have been summarised in Table 1 .

Twenty patients having desk jobs resumed their previous employment within 2 weeks, and remaining by 5 weeks.
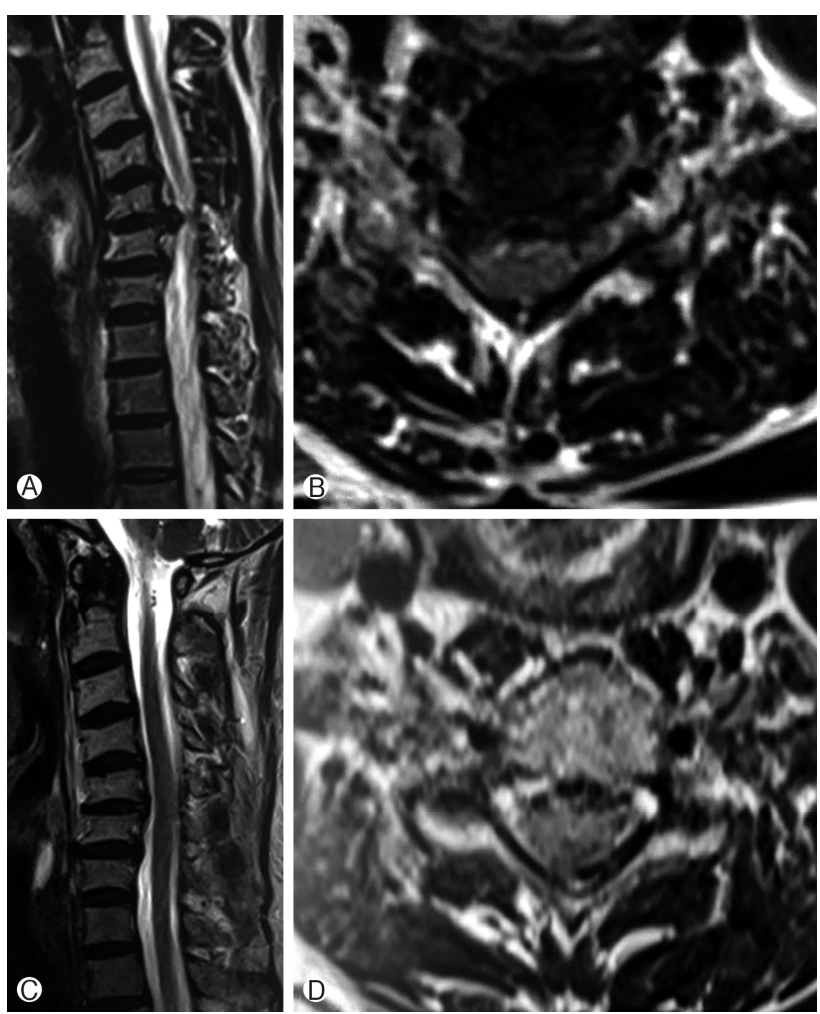

Fig. 5. (A) Pre-op sagittal, (B) Pre-op axial, (C) Post-op sagittal, (D) Post-op axial.

Table 1. Summarising results

\begin{tabular}{lccc}
\hline \hline Parameter & Pre-operative & Final follow up & $\mathrm{p}$-value \\
\hline VAS-neck pain & $6.2 \pm 0.72$ & $1.67 \pm 0.59$ & 0.00001 \\
VAS- arm pain & $7.25 \pm 0.71$ & $1.5 \pm 061$ & 0.00001 \\
$\mathrm{NDI}$ & $64.7 \pm 7.62$ & $13.48 \pm 5.42$ & 0.00001 \\
Disc height & $6.15 \mathrm{~mm}$ & $5.24 \mathrm{~mm}$ & 0.00001 \\
Cervical angle & $13.93 \pm 3.7$ & $15.58 \pm 6.66$ & 0.060 \\
Focal angle od involved disc & $0.86 \pm 1.66$ & $1.7 \pm 1.81$ & 0.0067 \\
\hline
\end{tabular}


Thirty patients were satisfied with the procedure .One patient was unsatisfied as he had persistent symptoms after surgery [Pre-operative VAS for arm pain and neck pain was 7 and 5, Post-operative it was 6 and 5] probably due to incomplete decompression. He was operated with ACDF two months after anterior PECD. No patient required delayed fusion procedure.

There were no complications like mortality, post-operative infection, neurological deterioration, hematoma formation, dysphagia, CSF leak, spontaneous fusion.

\section{DISCUSSION}

This study presents results of 31 patients treated with anterior PECD which shows fall of VAS and NDI after surgery. Cervical lordosis angle is well maintained with $0.91 \mathrm{~mm}$ fall in disc height of operated level post-surgery.

Surgeons strive to achieve better results day by day and try new techniques, which drives them to incorporate minimally invasive surgery ${ }^{10)}$. Success of percutaneous endoscopic lumbar discectomy has encouraged surgeons to perform percutaneous endoscopic decompression for cervical disc herniations ${ }^{11-13)}$. Minimally invasive cervical disc surgery has evolved from procedures like percutaneous fluoroscopy guided disc decompression, automated nucleotomy ${ }^{14)}$, percutaneous cervical disc decompression using chemonucleosis ${ }^{15)}$, percutaneous laser decompression $^{12,16)}$. But these were blind procedures without direct visualisation. In today's era development of cervical endoscope has improved fineness of percutaneous cervical disc surgery due to direct visualisation.

When we go through literature we find that majority of improvement in cervical disc surgery resolves around development of newer implants to be inserted in empty disc space after discectomy. There is lack of clarity about sufficiency of anterior cervical decompression without fusion [ACD]. Whether fusion is really necessary to preserve disc space height and whether disc height maintenance is important for good clinical outcome is unclear $^{3-5,11,14)}$.

Total volume of cervical disc is much less than that of lumbar disc. Cervical nerve root is confined to a smaller space than lumbar nerve root, therefore, a small volume reduction in cervical disc herniation produces a much greater effect ${ }^{17)}$.

Yong et al. ${ }^{16)}$ studied 36 patients treated with anterior percutaneous cervical discectomy. They found that mean disc height reduction after anterior PECD was $0.8 \mathrm{~mm}$ after 24 months follow up. In a study by Ruetten et al. ${ }^{18)}$ mean disc height reduction after anterior endoscopic cervical discectomy was $1.1 \mathrm{~mm}$ after 24 months follow up, while Yadav et al. ${ }^{19)}$ found that disc height reduction was $1.1 \mathrm{~mm}$ after anterior PECD. In our cases mean disc height reduction was $0.91 \mathrm{~mm}$ after 28.5 months of follow up period.

Incidence of spontaneous fusion in ACD is $4 \%$ to $74 \% \%^{5,20-22)}$. Although there is reduction in disc height complete disc space collapse and fusion is not reported after anterior PECD ${ }^{16)}$.

In percutaneous cervical endoscopic discectomies done by Ahn $Y$ et al. ${ }^{16)}$ VAS reduced from $8.78 \pm 1.6$ pre-operative to
$2.75 \pm 2.5$ post-operatively. In a study by Parihar et al. ${ }^{23)}$ VAS for arm and neck pain improved from 6.7 and 3.2 to 1.7 and 1.1 respectively. In our study VAS for neck pain was $6.2 \pm 0.72$ and at final follow up it was $1.67 \pm 0.59$ and VAS for radicular upper limb pain was 7.25 \pm 0.71 and at final follow up was 1.5 \pm 0.61 .

Even though there is disc height reduction, VAS and NDI show significant fall in our study which shows that there is no relationship between disc height reduction and clinical outcome, as shown by many other studies too $16,17,23)$.

It has been found that sizable numbers of patients develop cervical alignment disruption after open ACD. In a study by Laing et al. ${ }^{24)}$ postoperative loss of overall lordosis was found in $54.5 \%$ of patients and segmental kyphosis was seen in $32.7 \%$ of patients. There is evidence that immediate loss of lordosis after open ACD is corrected with time but still 10-20\% patients remain kyphotic. In a study by Yong et al. ${ }^{16)}$ pre-operative overall cervical lordosis was $15.78^{\circ} \pm 8.01^{\circ}$ which post-operatively became $16.00^{\circ} \pm 8.06^{\circ}$ and focal lordotic angle of treated level preoperative was $0.36^{\circ} \pm 4.29^{\circ}$ which improved to $2.33^{\circ} \pm 3.55^{\circ}$. Yadav et al. ${ }^{19)}$ found that mean pre-operative lordotic angle changed from $6.3^{\circ}$ to $6.1^{\circ}$ after surgery and mean focal angle of involved segment changed from $2.1^{\circ}$ to $2.0^{\circ}$.

In our study this change of cervical angle was from $13.93^{\circ} \pm$ $3.73^{\circ}$ pre operatively to $15.98^{\circ} \pm 6.66^{\circ}$ post-operatively. Thus, overall cervical lordosis was well maintained. This change is not statistically significant [p-value 0.06]. Focal lordotic angle of treated level increased from $0.83^{\circ} \pm 1.66^{\circ}$ to $1.7^{\circ} \pm 1.81^{\circ}$ which is statistically significant with $p$-value 0.0067 . The cause for maintenance of lordotic angle can be preservation of anterior structures like longus coli, anterior longitudinal ligament, and anterior annulus, most of the anterior and central disc and selective removal of herniated posterior disc. There is selective reduction posterior disc space, thus, maintaining lordosis 24.

In study by Rueten et al. ${ }^{18)}$ revision rate was $7.4 \%$, while in study by Parihar et al. ${ }^{23)}$ was $0.95 \%$. In our study it was $3.2 \%$. Stringent selection criteria and smaller sample size may be reasons for better results in our cases.

It has been reported that there is 3 times more risk of adjacent disc degeneration in incorrectly marked disc during $A C D F^{25}$. This might be discouraging for endoscopic surgeons. But Hadley et al $^{26)}$ reported that in carefully selected patients only $1.5 \%$ patients need delayed fusion procedure. As stated by many studies, there is lack of correlation between disc height loss and symptoms. Thus inserting expensive implant or donor site morbidity due to bone grafting might be overdone for many cases.

Endoscopic cervical disc surgeries have longer learning curve and its application is limited to disc surgery for now. It is not recommended for advanced spondylosis with severe disc space narrowing. The success rate of anterior PECD is reported to be ranging from $51 \%$ to $94.5 \% 3,13,15,17$ )

Though PECD is rewarding, scientific evidence for this procedure is low; few randomised control trials [RCTs] are available evaluating its effectiveness.

Limitations of this study are no comparative group, fewer number of patients short period of follow up. More studies 
with higher number of patients with longer follow up and randomisation are needed to shed more light on effectiveness of anterior PECD.

\section{CONCLUSION}

Anterior PECD is safe and effective alternative to ACDF in properly selected cases. It has less approach related morbidity leading to lesser hospital stay and faster recovery. More studies with randomisation are needed to improve our knowledge about this procedure.

\section{Conflict of Interest}

Authors declare that there is no conflict of interest.

\section{REFERENCES}

1. Bohlman H, Emery S, Goodfellow D, Jones P: Robinsons anterior cervical discectomy and arthrodesis for cervical radiculopathy. JBJS 75-A(9):1298-1307, 1993

2. Gore D, Sepic S: Anterior cervical discectomy and fusion for painful cervical disc disease. SPINE 23(19):2047-2051, 1998

3. Dowd G, Fremont P: Anterior cervical discectomy is fusion necessary? J Neurosurg 90(Spine 1):8-12, 1999

4. Martins A: Anterior cervical discectomy with and without interbody bone graft. J Neurosurg 44:290-295, 1976

5. Pointillart V, Cernier A, Vital J, Senegas J: Anterior discectomy without interbody fusion for cervical disc herniation. Eur Spine J 4:45-51, 1995

6. Lee S H, Lee J H, Choi W C, Jung B, Mehta R: Anterior minimally invasive approaches for the cervical spine. Orthop Clin North Am 3:327-337, 2007

7. Nadkarni S, Kohli P, Patel B, Gore S, Kalkarni B: STITCHLESS percutaneous endoscopic cervical discectomy, are we moving towards day care discectomy procedure? Indian J Orthop 51: 653-657, 2017

8. Khiste S,Tari R: Platelet-rich fibrin as a biofuel for tissue regeneration. ISRN Biomatrials 1-6, 2013

9. Kuffler D: Platelet-rich plasma and the elimination of neuropathic pain. Mol Neurobiol 48:315-332, 2013

10. Kulkarni A, Ruparel S: How to incorporate minimally invasive spine surgery in practice? Journal of Minimally Invasive Spine Surgery and Technique 3(1):9-12, 2018

11. Ahn Y, Lee S H, Lee S C, Shin S, Chung S: Factors predicting excellent outcome of percutaneous cervical discectomy?: analysis of 111 consecutive cases. Neuroradiology 46:378-384, 2004

12. Hellinger J: Technical aspects of the percutaneous cervical and lumbar laser-disc-decompression and nucleotomy. Neurological Research 21(1):99-102, 1999

13. Knight M, Goswami A, Patko J: Cervical percutaneous laser disc decompression?: preliminary results of an ongoing prospective outcome Study. Journal of Clinical Laser Medicine \& Surgery 19(1):3-8, 2001

14. Bonaldi G, Minonzio G, Belloni G, Dorizzi A, Fachinetti P, Marra A: Percutaneous cervical diskectomy?: preliminary experience. Neuroradiology 36:483-486, 1994.

15. Hoogland T: Scheckenbach low-dose chemonucleolysis combined with percutaneous nucleotomy in herniated cervical disks. J of Spinal Disorders 8(3):228-232, 1995

16. Ahn Y, Lee S H, Shin S W: Percutaneous endoscopic cervical discectomy?: clinical outcome and radiographic changes. Photomedicine and Laser Surgery 23(4):362-368, 2005

17. Ahn Y: Percutaneous endoscopic cervical discectomy using working channel endoscopes. Expert Review of Medical Devices 1745-2422, 2016

18. Ruetten S, Komp M, Merk H: Full-endoscopic anterior decompression versus conventional anterior decompression and fusion in cervical disc herniations. International Orthopaedics (SICOT) 33:1677-1682, 2009

19. Yadav Y, Parihar V, Ratre S, Kher Y: Endoscopic anterior decompression in cervical disc disease. Neurology India 62(4): 417-422, 2014

20. Snyder G, Bernhardt M: Anterior cervical fractional interspace decompression for treatment of cervical radiculopathy: a review of the first 66 cases. Clin Ortho and Related Research 246:9299, 1989

21. Grisoli F, Graziani N, Fabrini A, Peragut J, Vincentelli F, Vasquez P: Anterior discectomy without fusion for treatment of lateral soft disc extrusion: a follow up of 120 cases. Neurosurgery 24(6):853-858, 1989

22. Husag L, Probst C: Microsurgical anterior approach to cervical discs * Review of 60 consecutive cases of discectomy without fusion. Acta Neurochirurgica 73:229-242, 1984

23. Parihar V, Yadav N, Ratre S, Dubey A, Yadav Y: Endoscopic anterior approach for cervical disc disease (disc preserving surgery). World Neurosurg 4:107-117, 2018

24. Laing R, Ng I, Seeley H, Hutchinson P: Prospective study of clinical and radiological outcome after anterior cervical discectomy. British Journal of Neurosurgery 15(4):319-323, 2001

25. Nassr A, Lee J, Bashir R, Rihn J, Eck J, Kang J, et al: Does incorrect level needle localization during anterior cervical discectomy and fusion lead to accelerated disc degeneration? SPINE 34(2):189-192, 2009

26. Hadley M, Sonntag V: Cervical disc herniations. The anterior approach to symptomatic interspace pathology. Neurosurg Clin N Am 4(1):45-52, 1993 